\title{
ESTUDO CINÉTICO DA HIDRÓLISE DO ACETATO DE ETILA EM MEIO ALCALINO EM REATOR BATELADA DE TANQUE AGITADO
}

\author{
M. S. de SOUZA ${ }^{1}$, V. M. V. CRUZ ${ }^{1}$, G. L. PENA ${ }^{1}$, A. B. de CARVALHO ${ }^{1}$, D. N. DANTAS ${ }^{1}$ \\ ${ }^{1}$ Centro Universitário do Leste de Minas Gerais, Departamento de Engenharia Química \\ E-mail para contato: mellyssa95@gmail.com
}

\begin{abstract}
RESUMO - A hidrólise é uma reação química em que ocorre a quebra de uma molécula por água, e inclui, na química orgânica, a reação em que utiliza-se um álcali ou um ácido no lugar da água. A hidrólise do acetato de etila foi realizada em meio alcalino, utilizando $\mathrm{NaOH}$, à temperatura de 22,35 e $45^{\circ} \mathrm{C}$, e para determinação da concentração do acetato de etila efetuou-se a titulação de uma alíquota da mistura reacional adicionando-a a $\mathrm{HCl}$ e titulando o excesso com $\mathrm{NaOH}$. Os dados experimentais foram ajustados às leis de velocidade de ordem zero, um e dois, utilizando o método integral. $\mathrm{O}$ melhor ajuste encontrado foi para a lei de velocidade de segunda ordem, a partir da qual determinou-se as constantes de velocidade (k), e a dependência da mesma com a temperatura, através da equação de Arrehnius. Os valores de k variaram de 20,6 a 33,2 L/mol.min. Determinou-se ainda a energia de ativação $\left(\mathrm{E}_{\mathrm{A}}\right)$ e o fator de frequência $\left(\mathrm{k}_{0}\right)$, sendo os valores encontrados de $1,7855 \times 10^{4} \mathrm{~J} / \mathrm{mol}$ para $\mathrm{E}_{\mathrm{A}}$, e $1,5313 \times 10^{6} \mathrm{~L} / \mathrm{mol}$.min para $\mathrm{k}_{0}$, estando ambos valores na faixa reportada na literatura.
\end{abstract}

\section{INTRODUÇÃO}

A hidrólise é um termo aplicado a reações orgânicas e inorgânicas que ocorrem em meio aquoso, em que a água presente efetua uma dupla troca com outro composto: um hidrogênio da molécula de água é transferido para um dos produtos, e o grupo $\mathrm{OH}^{-}$é transferido para o outro produto. Por conveniência, têm sido consideradas como hidrólise, a reação onde um álcali é utilizado no lugar da água, obtendo um sal alcalino de um ácido no final (HIJAZIN et al., 2010).

A cinética química representa o estudo da velocidade das reações, de como a velocidade varia em função das diferentes condições e quais os mecanismos de desenvolvimento de uma reação (LAVORENTI, 2002).

Ésteres são compostos orgânicos que possuem a fórmula geral RCOOR', onde um grupo carbonila está ligado a um grupo alcoxila (-OR). Os ésteres podem sofrer hidrólise ácida ou alcalina, sendo a hidrólise alcalina comumente chamada de saponificação. Refluxando um éster com hidróxido de sódio $(\mathrm{NaOH})$ aquoso, por exemplo, produz-se um álcool e um sal de sódio (SOLOMONS e FRYHLE, 2002). A reação de hidrólise do acetato de etila em meio básico (Equação 1) é aparentemente simples, porém somente um estudo aprofundado do comportamento reacional poderá confirmar a cinética da reação.

$$
\mathrm{CH}_{3} \mathrm{COOCH}_{2} \mathrm{CH}_{3}+\mathrm{NaOH} \rightarrow \mathrm{CH}_{3} \mathrm{COONa}+\mathrm{CH}_{3} \mathrm{CH}_{2} \mathrm{OH}
$$

A hidrólise alcalina de um éster é uma reação não elementar, pois reações elementares envolvem apenas uma etapa. $\mathrm{O}$ conjunto de todas as etapas de reação é denominado mecanismo 
de reação. Em geral, uma das etapas é lenta e as demais são rápidas. A etapa lenta determina a lei de velocidade e a ordem da reação (FOGLER, 2012; SOUZA e FARIAS, 2008).

Utilizou-se um reator batelada de tanque agitado, operando isotermicamente, a volume constante, para obtenção dos dados experimentais relativos a reação de hidrólise do acetato de etila em meio alcalino. Este trabalho avaliou a progressão da reação de hidrólise ao longo do tempo e, a partir dos dados experimentais obtidos, determinou-se os parâmetros cinéticos como a velocidade específica, ordem de reação, bem como as respectivas equações de taxa a três temperaturas distintas: 22,35 e $45^{\circ} \mathrm{C}$. Além disso, determinou-se a energia de ativação e o fator de frequência bem como demonstrou-se as discussões inerentes aos resultados experimentais.

\section{MATERIAIS E MÉTODOS}

Adicionou-se a béqueres de $250 \mathrm{~mL}, 250 \mathrm{~mL}$ de solução de acetato de etila $0,02 \mathrm{M}$, e 250 $\mathrm{mL}$ de solução de hidróxido de sódio $0,02 \mathrm{M}$. Efetuou-se o procedimento a três temperaturas, de 22,35 e $45^{\circ} \mathrm{C}$, monitoradas com o auxílio de termômetros. O aquecimento foi realizado com a utilização de banho termostático. Quando as soluções atingiram as temperaturas desejadas, foram misturadas rapidamente em reatores (béqueres de $1000 \mathrm{~mL}$ ) e mantidas a agitação e temperatura constantes, através da utilização de manta aquecedora com agitação.

Imediatamente após efetuar a mistura das soluções nos reatores, acionou-se o cronômetro, retirando-se alíquotas de $10 \mathrm{~mL}$ da mistura, nos tempos $0,5,10,15,20,25$ e 30 minutos. As alíquotas foram adicionadas a erlenmeyers de $250 \mathrm{~mL}$, aos quais foi previamente adicionado $30 \mathrm{~mL}$ de solução de ácido clorídrico $0,01 \mathrm{M}$ e 4 gotas de fenolftaleína. Em seguida a adição das alíquotas aos erlenmeyers, efetuou-se rapidamente a titulação das mesmas com hidróxido de sódio $0,01 \mathrm{M}$, até que as mesmas atingissem o ponto de equivalência, indicado pela fenolftaleína adicionada. $\mathrm{O}$ volume de hidróxido de sódio consumido foi anotado.

Após a obtenção dos dados experimentais, calculou-se a concentração de acetato de etila em cada tempo, utilizando a Equação 2, de modo que a variação da concentração permitiu determinar a ordem da reação, através do método integral de ajuste às leis de velocidade.

$$
[\text { Acetato de etila }]=\frac{\left([\mathrm{HCl}]_{\mathrm{adc}} \times \mathrm{V}_{\mathrm{HCl} \mathrm{adc}} \times \mathrm{Fc}_{\mathrm{HCl}}\right)-\left([\mathrm{NaOH}]_{\mathrm{tit}} \times \mathrm{V}_{\mathrm{NaOH} \mathrm{tit}} \times \mathrm{Fc}_{\mathrm{NaOH} \text { tit }}\right)}{\mathrm{V}_{\text {aliquota }}}
$$

Para estudar corretamente a velocidade da reação e demais parâmetros cinéticos, é necessário determinar primeiramente a ordem da reação, sendo a ordem da reação dada pela soma dos expoentes das concentrações (ordens parciais), na lei de velocidade. A ordem da reação não pode ser deduzida da estequiometria da reação global, mas sim dos dados experimentais de velocidade de reação, bem como do mecanismo da reação (SOUZA e FARIAS, 2008). A equação da taxa genérica de consumo do reagente $A$, em uma reação em fase líquida, com volume constante, $\mathrm{A}+\mathrm{B} \rightarrow$ Produtos, pode ser escrita conforme Equação 3:

$$
\left(-\mathrm{r}_{\mathrm{A}}\right)=-\frac{\mathrm{d}[\mathrm{A}]}{\mathrm{dt}}=\mathrm{k} \cdot[\mathrm{A}]^{\alpha} \cdot[\mathrm{B}]^{\beta}
$$

Para determinar a ordem de reação pelo método integral, supõe-se uma ordem de reação e integra-se a equação diferencial utilizada para modelar o sistema em batelada. Se a suposta ordem de reação for correta, o gráfico dos dados de concentração-tempo deve ser linear (FOGLER, 2012). A reação é de ordem zero quando a velocidade da reação química é 
independente da concentração do reagente (Equação 4), obedecendo a lei de velocidade resultante da integração de ambos os termos (Equação 5), dada na Equação 6:

$$
\begin{aligned}
& -\frac{\mathrm{d}[\mathrm{A}]}{\mathrm{dt}}=\mathrm{k} \cdot[\mathrm{A}]^{0}=\mathrm{k} \\
& -\int_{[\mathrm{A}]_{0}}^{[\mathrm{A}]} \mathrm{d}[\mathrm{A}]=\mathrm{k} \int_{0}^{\mathrm{t}} \mathrm{dt} \\
& -[\mathrm{A}]+[\mathrm{A}]_{0}=\mathrm{k} \cdot \mathrm{t}
\end{aligned}
$$

Reações de primeira ordem são aquelas nas quais a velocidade da reação química é proporcional à concentração de um reagente (Equação 7). A lei da velocidade de primeira ordem é uma das formas mais comuns da lei da velocidade (LAVORENTI, 2002), e é dada pela integração de ambos os termos (Equação 8), resultando na Equação 9:

$$
\begin{gathered}
-\frac{\mathrm{d}[\mathrm{A}]}{\mathrm{dt}}=\mathrm{k} \cdot[\mathrm{A}]^{1}=\mathrm{k} \cdot[\mathrm{A}] \\
-\int_{[\mathrm{A}]_{0}}^{[\mathrm{A}]} \frac{\mathrm{d}[\mathrm{A}]}{[\mathrm{A}]}=\mathrm{k} \int_{0}^{\mathrm{t}} \mathrm{dt} \\
-\ln \left(\frac{[\mathrm{A}]}{[\mathrm{A}]_{0}}\right)=\mathrm{k} \cdot \mathrm{t}
\end{gathered}
$$

Reações de segunda ordem são aquelas nas quais a velocidade da reação é proporcional ao produto das concentrações de dois reagentes. Leis da velocidade de segunda ordem envolvem dois reagentes, e para ambos a concentração depende do tempo (LAVORENTI, 2002). Para esta lei de velocidade, assumiu-se que a concentração do acetato de etila (A) e do hidróxido de sódio (B) variam igualmente com o tempo, sendo estes componentes adicionados em quantidades equimolares e sendo a estequiometria dos mesmos proporcional. Assim, utilizou-se $[\mathrm{A}]=[\mathrm{B}]$. A Equação 10 descreve a lei da velocidade de segunda ordem, na qual a integração de ambos os termos (Equação 11) resulta na Equação 12:

$$
\begin{gathered}
-\frac{\mathrm{d}[\mathrm{A}]}{\mathrm{dt}}=\mathrm{k} \cdot[\mathrm{A}]^{\alpha} \cdot[\mathrm{B}]^{\beta}=\mathrm{k} \cdot[\mathrm{A}]^{2} \\
-\int_{[\mathrm{A}]_{0}}^{[\mathrm{A}]} \frac{\mathrm{d}[\mathrm{A}]}{[\mathrm{A}]^{2}}=\mathrm{k} \int_{0}^{\mathrm{t}} \mathrm{dt} \\
\frac{1}{[\mathrm{~A}]}-\frac{1}{[\mathrm{~A}]_{0}}=\mathrm{k} \cdot \mathrm{t}
\end{gathered}
$$

Determinou-se a constante de velocidade das reações, através do coeficiente angular do ajuste linear, e a dependência da mesma com a temperatura, através da equação de Arrehnius linearizada (Equação 13). Conhecendo a constante de velocidade a diferentes temperaturas, foi possível determinar a energia de ativação $\left(\mathrm{E}_{\mathrm{A}}\right)$ e o fator de frequência $\left(\mathrm{k}_{0}\right)$ da reação.

$$
\ln (\mathrm{k})=\ln \left(\mathrm{k}_{0}\right)-\frac{\mathrm{E}_{\mathrm{A}}}{\mathrm{R}} \frac{1}{\mathrm{~T}}
$$


Os cálculos e ajustes lineares foram efetuados utilizando o software Origin ${ }^{\circledR} 8$.

\section{RESULTADOS E DISCUSSÃO}

A partir das leis de velocidade presentes nas Equações 6, 9 e 12 e conhecendo a concentração de acetato de atila em cada instante, calculada através da Equação 2, realizou-se os cálculos necessários e o ajuste linear dos dados, cujos gráficos estão presentes na Figura 1.

Figura 1 - Ajuste linear dos dados experimentais a lei de velocidade de primeira ordem
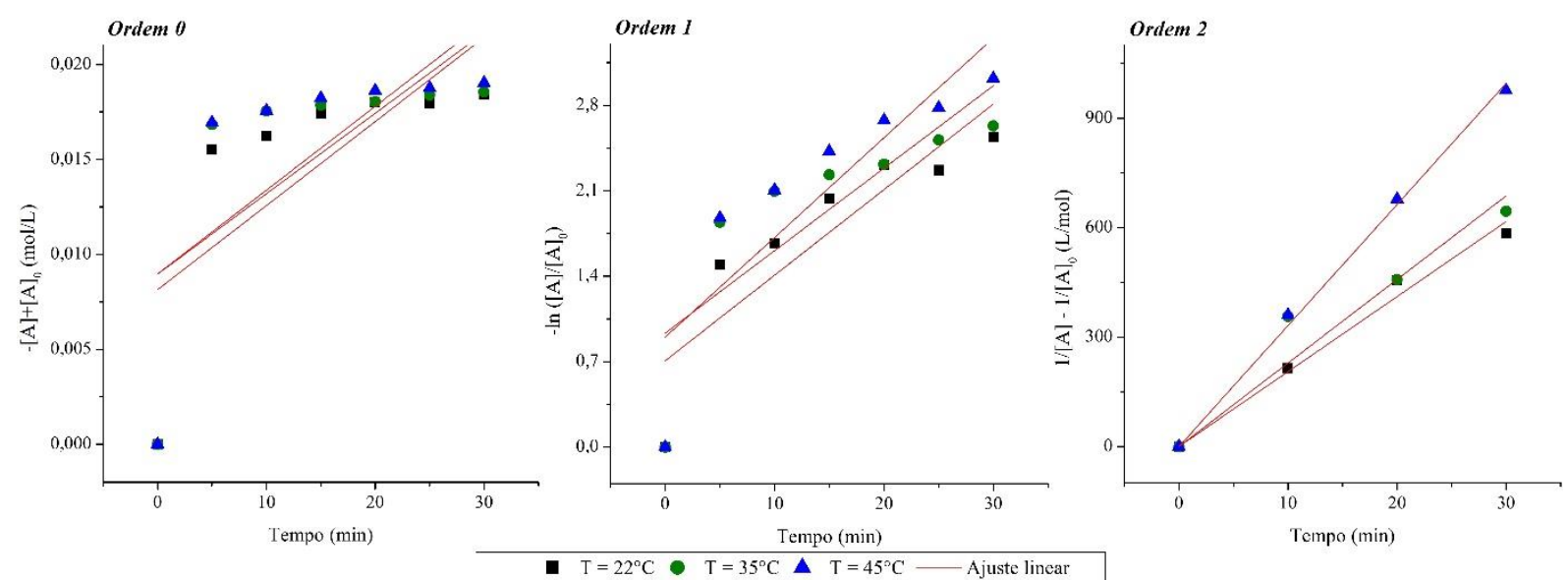

As equações encontradas para cada ajuste, bem como as médias do coeficiente de determinação nas variadas temperaturas, estão presentes na Tabela 1:

Tabela 1 - Ajuste linear dos dados experimentais

\begin{tabular}{ccccc}
\hline Ordem & Temperatura $\left({ }^{\circ} \mathbf{C}\right)$ & Ajuste linear & Coeficiente de determinação $\left(\mathbf{R}^{2}\right)$ & Média do $\mathbf{R}^{\mathbf{2}}$ \\
\hline \multirow{2}{*}{0} & 22 & $\mathrm{y}=4,4232 \times 10^{-4} \mathrm{x}+0,00815$ & 0,42833 & \\
& 35 & $\mathrm{y}=4,2340 \times 10^{-4} \mathrm{x}+0,00896$ & 0,34647 & 0,38171 \\
& 45 & $\mathrm{y}=4,4128 \times 10^{-4} \mathrm{x}+0,00897$ & 0,37033 & \\
\hline \multirow{2}{*}{1} & 22 & $\mathrm{y}=0,07014 \mathrm{x}+0,70729$ & 0,73349 & 0,67730 \\
& 35 & $\mathrm{y}=0,06760 \mathrm{x}+0,93386$ & 0,59395 & \\
& 45 & $\mathrm{y}=0,08175 \mathrm{x}+0,90043$ & 0,70446 & 0,98667 \\
2 & 22 & $\mathrm{y}=20,55669 \mathrm{x}$ & 0,99320 & \\
\hline & 35 & $\mathrm{y}=25,90646 \mathrm{x}$ & 0,96799 & 0,99881 \\
\hline
\end{tabular}

A partir da Figura 1 observa-se que o ajuste dos dados experimentais às leis de velocidade de ordem 0 e 1 não possuem comportamento linear. Já para o ajuste de segunda ordem, observase comportamento linear satisfatório, com coeficiente de determinação $\left(\mathrm{R}^{2}\right)$ médio de 0,98667 , possibilitando afirmar que a hidrólise do acetato de etila em meio alcalino é uma reação de $2^{\mathrm{a}}$ ordem. Para afirmar que a reação é de determinada ordem através do método integral, sabe-se que é desejável um $\mathrm{R}^{2}$ mais próximo da unidade, contudo, o desvio se justifica principalmente por erros devido ao aparato experimental ter se dado de forma completamente manual.

A partir da Equação 12 sabe-se que a constante de velocidade (k) é o coeficiente angular das equações determinadas para cada reta. Conhecendo $\mathrm{k}$, as equações de taxa da reação em cada temperatura puderam então ser determinadas, e estão presentes na Tabela 2: 


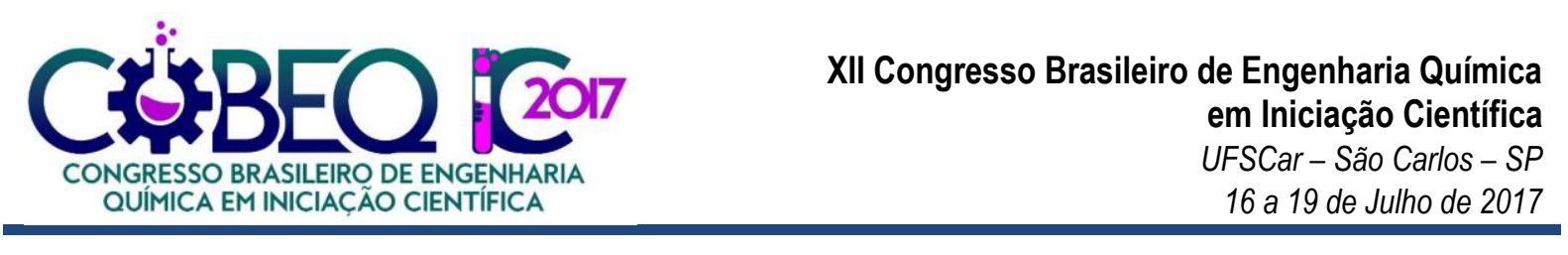

Tabela 2 - Constante de velocidade e equações de taxa da reação

\begin{tabular}{cccc}
\hline Ordem & Temperatura $\left({ }^{\circ} \mathbf{C}\right)$ & $\mathbf{k}(\mathbf{L} / \mathbf{m o l} . \mathbf{m i n})$ & Equação da taxa \\
\hline \multirow{2}{*}{2} & 22 & 20,55669 & $\left(-\mathrm{r}_{\mathrm{A}}\right)=20,55669[\mathrm{~A}]^{2}$ \\
& 35 & 25,90646 & $\left(-\mathrm{r}_{\mathrm{A}}\right)=25,90646[\mathrm{~A}]^{2}$ \\
& 45 & 33,18541 & $\left(-\mathrm{r}_{\mathrm{A}}\right)=33,18541[\mathrm{~A}]^{2}$ \\
\hline
\end{tabular}

Analisando os dados da Tabela 2, nota-se que houve um aumento da constante de velocidade com o aumento da temperatura do meio reacional. A dependência da constante de velocidade com a temperatura é dada pela equação de Arrhenius (Equação 13), uma equação empírica, mas com suporte teórico (SOUZA e FARIAS, 2008). Realizou-se o ajuste gráfico de ln (k) versus 1/T, presente na Figura 2, a partir da Equação 13 e dos dados da Tabela 3, sendo possível assim determinar o fator de frequência $\left(\mathrm{k}_{0}\right)$ e a energia de ativação $\left(\mathrm{E}_{\mathrm{A}}\right)$ :

Tabela 3 - Cálculos para o ajuste linear dos dados experimentais a Lei de Arrehnius

\begin{tabular}{ccccc}
\hline Temperatura $\left({ }^{\circ} \mathbf{C}\right)$ & Temperatura $(\mathbf{K})$ & $\mathbf{k}(\mathbf{L} / \mathbf{m o l} . \mathbf{m i n})$ & $\mathbf{1} / \mathbf{T}\left(\mathbf{K}^{-\mathbf{1}}\right)$ & $\ln (\mathbf{K})$ \\
\hline 22 & 295,15 & 20,55669 & 0,003388 & 3,02319 \\
35 & 308,15 & 25,90646 & 0,003245 & 3,25449 \\
45 & 318,15 & 33,18541 & 0,003143 & 3,50211 \\
\hline
\end{tabular}

Sendo a equação da reta a expressa na Equação 14, e sendo a constante universal dos gases $(\mathrm{R})$ igual a 8,314462 J/mol.K, determinou-se $\mathrm{k}_{0}$ e $\mathrm{E}_{\mathrm{A}}$, conforme Equações 15 e 16:

$$
\begin{gathered}
\mathrm{y}=-2.147,48284+14,24161 \\
-\frac{\mathrm{E}_{\mathrm{A}}}{\mathrm{R}}=-2.147,48284 \rightarrow \mathrm{E}_{\mathrm{A}}=1,7855 \times 10^{4} \mathrm{~J} / \mathrm{mol} \\
\ln \left(\mathrm{k}_{0}\right)=14,24161 \rightarrow \mathrm{k}_{0}=1,5313 \times 10^{6} \mathrm{~L} / \mathrm{mol} . \mathrm{min}
\end{gathered}
$$

Figura 2 - Ajuste linear dos dados experimentais a Lei de Arrehnius

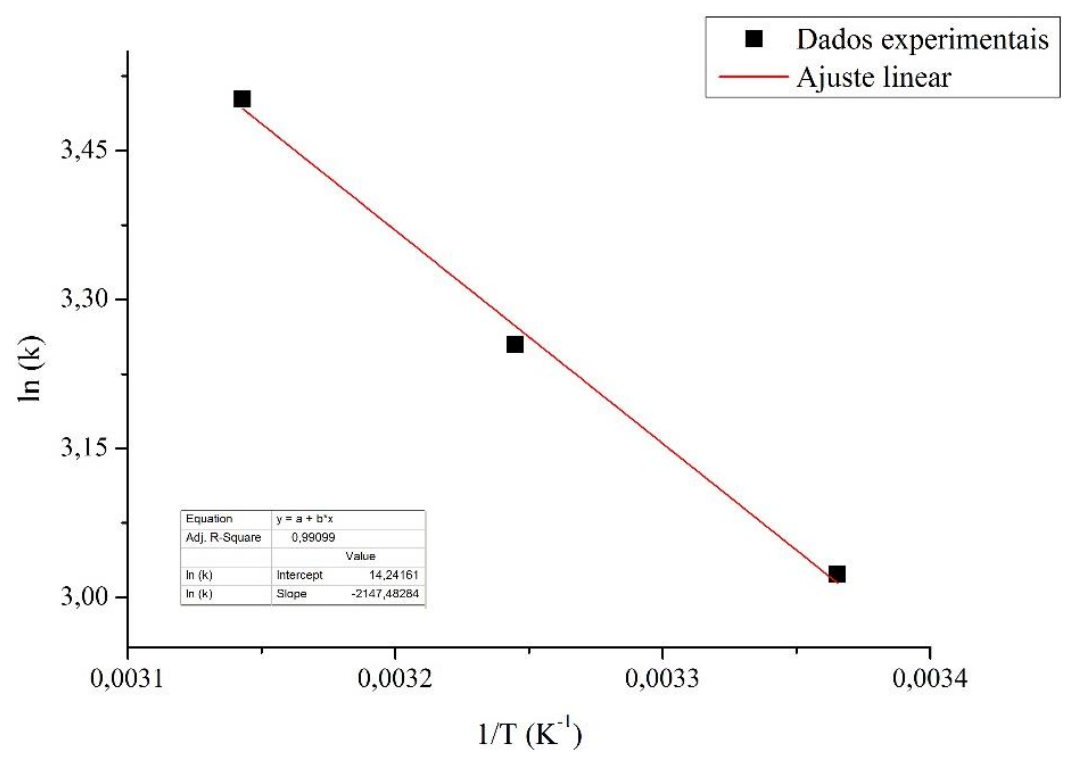

A energia de ativação $\left(E_{\mathrm{A}}\right)$ pode ser interpretada como uma barreira à transferência de energia entre as moléculas reagentes, a qual deve ser vencida. Reações com altos valores de $\mathrm{E}_{\mathrm{A}}$ são muito dependentes da temperatura, e reações com baixos valores de $\mathrm{E}_{\mathrm{A}}$ são relativamente 
independentes da temperatura (FOGLER, 2012; LEVENSPIEL, 2000). Souza e Farias (2008) afirmam que valores de $E_{\mathrm{A}}$ para a maioria das reações químicas são encontrados na faixa de 0 a $33,0 \times 10^{4} \mathrm{~J} / \mathrm{mol}$, estando o valor encontrado, de $1,7855 \times 10^{4} \mathrm{~J} / \mathrm{mol}$, contido na faixa citada.

O fator de frequência $\left(\mathrm{k}_{0}\right)$ representa um fator proporcional ao número de colisões, e pode ser considerado aproximadamente constante, dependente apenas da probabilidade de colisão das moléculas e da temperatura (SCHMAL, 2010). De acordo com Souza e Farias (2008), valores de $\mathrm{k}_{0}$ são encontrados na faixa de $10^{6}$ a $10^{10} \mathrm{~L} / \mathrm{mol}$.min para reações bimoleculares, estando o valor encontrado, de $1,5313 \times 10^{6} \mathrm{~L} / \mathrm{mol}$.min, de acordo com a faixa esperada.

Sendo o $\mathrm{R}^{2}$ do ajuste linear igual a 0,99099, o desvio justifica-se pelo fato de reações não elementares serem constituídas por várias etapas de reações elementares, sendo assim, a ocorrência de etapas intermediárias leva a uma mudança na energia de ativação, gerando um ajuste não-linear. Cada etapa do mecanismo tem uma energia de barreira diferente, sendo a energia da equação global um somatório de todas as energias envolvidas (SCHMAL, 2010).

\section{CONCLUSÕES}

A ordem da reação de hidrólise do acetato de etila em meio alcalino foi determinada através do método integral. Tal método baseia-se na suposição de uma ordem e análise a partir da integração e regressão linear dos dados. Através dos cálculos efetuados, pode-se concluir que a hidrólise do acetato de etila em meio alcalino é uma reação de segunda ordem, para a qual o ajuste dos dados experimentais a lei de velocidade apresentou comportamento linear, requisito para afirmar que uma reação é de determinada ordem pelo método integral.

O método integral permitiu ainda calcular a constante de velocidade da reação a diferentes temperaturas. A partir do cálculo, observou-se que a mesma apresenta dependência direta da temperatura, conforme o esperado através do conhecimento teórico. O ajuste linear dos dados a lei de Arrehnius possibilitou calcular a energia de ativação e o fator de frequência desta reação. Os valores determinados experimentalmente encontram-se dentro da faixa citada na literatura.

\section{REFERÊNCIAS}

FOGLER, H. S. Elementos de engenharia das reações químicas. 4. ed. Rio de Janeiro: LTC, 2012. 888 p.

HIJAZIN, C. A. H.; SIMÕES, A. T.; SILVEIRA, D. R. Hidrólise ácida, alcalina e enzimática. 2010. Revista Atitude. Ano IV, v.4, n.7, p.89-83, jan-jun., 2010.

LAVORENTI, A. Cinética Química. Piracicaba: Universidade Estadual de São Paulo, 2002.

LEVENSPIEL, O. Engenharia das reações químicas. 3. ed. São Paulo: Editora Blucher, 2000.

SCHMAL, M. Cinética e reatores: Aplicação na engenharia química. 1. ed. Rio de Janeiro: Synergia, 2010. $678 \mathrm{p}$.

SOLOMONS, T. W. G.; FRYHLE, C. B. Química Orgânica. 7. ed. Rio de Janeiro: LTC, 2002. v.2. 474 p.

SOUZA, A. A. de; FARIAS, R. F. de. Cinética química: Teoria e prática. 1. ed. Campinas: Editora Átomo, 2008. 88 p. 